\title{
Aromatic Force Field for Out-of-plane Molecular Vibrations
}

\author{
SVEN J. CYVIN, BJØRG N. CYVIN, JON BRUNVOLL and GRETHE NEERLAND
}

\author{
Division of Physical Chemistry, The University of Trondheim, N-7034 Trondheim-NTH, Norway
}

The out-of-plane molecular vibrations of aromatic molecules are considered. Two "classical" force field approximations are discussed in some detail. They are the Coulson-Senent force field with two parameters and that of Whiffen et al. with seven parameters. It is concluded that a new approximation would be desirable, simpler than the Whiffen force field and more accurate than the one of Coulson and Senent. Theoretical considerations based on the approach of Whiffen provided a guide for developing such an approximation. We were able to develop a useful force field containing three parameters in benzene and five parameters in naphthalene as well as any other condensed aromatic molecule. The applications to benzene and naphthalene are reported. The transferability of the force field to anthracene and pyrene is briefly mentioned.

The molecular vibrations of conjugated $\pi$-electron systems have been studied by a great number of investigators. For benzene in particular the studies go back to $1934{ }^{1,2}$ The present paper deals with an out-of-plane force field for benzene and condensed aromatic molecules. Two "classical" papers on this special problem date back to $1955^{3,4}$

The in-plane vibrations of condensed aromatics have been studied systematically by several researchers. A modified Urey-Bradley force field for benzene ${ }^{5}$ was extended by Scherer ${ }^{6}$ to condensed aromatics and applied to naphthalene. Califano ${ }^{7}$ extended the calculations to anthracene. His work was followed by a series of papers by Neto and Califano with collaborators. ${ }^{8-11}$ Additional work is due to Bree et al. ${ }^{12}$ More recently the problem was taken up again in our laboratory, ${ }^{13-17}$ independently of the studies by Ohno, ${ }^{18,19}$ which are related to the same problem.

In comparison to this large amount of work the out-of-plane vibrations of condensed aromatics have received much less attention. In the paper of Coulson and Senent ${ }^{4}$ the out-of-plane vibrations of ethylene, benzene and naphthalene are treated. The developed force field contains only two force constants. We agree that a small number of parameters is an essential virtue of the force field approximation. A critical examination of the Coulson-Senent force field shows, however, that it does not meet the modern standards of accuracy in view of more recent experimental evidence. Whiffen ${ }^{3}$ developed a seven-parameter force field with numerical force constants adjusted to the outof-plane frequencies for benzene. These parameters were applied to naphthalene by Scully and Whiffen. ${ }^{20,21}$ The force field approximation has also been applied to the out-of-plane vibrations of anthracene ${ }^{22}$ and pyrene. ${ }^{12}$ The Whiffen force field reproduces satisfactorily the experimental frequencies in the relatively few cases where it has been applied. However, it has some deficiencies of a principal nature: (a) The number of parameters should be decreased, if possible, within a reasonable degree of approximation. (b) The rules for application to an arbitrary condensed aromatic should be simpler. In the existing applications to naphthalene, ${ }^{20,21}$ anthracene ${ }^{22}$ and pyrene ${ }^{12}$ it seems necessary to introduce special conventions in special cases. If not directly ambiguous, the application to higher condensed aromatics would certainly not be simple, to say the least. In the first part ${ }^{13}$ of a series of papers on condensed aromatics from this laboratory a seven-parameter aromatic force field was suggested. As far as the out-of-plane vibrations are concerned the approximation contains only three parameters. Their values were adjusted to the experimental assignment of frequencies for naphthalene and seemed to be applicable to certain other condensed aromatics. The approximation was reported to fail 
for benzene, ${ }^{13}$ however. Further investigations showed it to be unsatisfactory as an aromatic force field in general.

There exist many more studies not reviewed here, dealing with the molecular force fields of aromatics, especially benzene and naphthalene, and including the out-of-plane modes of vibration as well as in-plane. The literature is too voluminous to be cited here. Beside that we are not concerned here with this part of the research unless it aims at the development of a force field transferable from one aromatic molecule to the other - in other words the development of an "aromatic force field".

From the above considerations we found the search for a new aromatic out-of-plane force field to be warranted. Such a force field should fulfil the following conditions. It should (a) be simple and contain as few parameters as possible, (b) be transferable to any condensed aromatic according to strict rules, (c) reproduce modern experimental frequencies satisfactorily and (d) be useful as an aid in the assignments of observed frequencies.

\section{THEORETICAL CONSIDERATIONS}

A theoretical analysis was performed on the basis of the work of Whiffen et al.,,20 but using the modern formalism in terms of the $T$ matrix. ${ }^{23}$ The analysis was found to add to the understanding of the main features of the Whiffen force field.

We have adopted the numbering of atoms in a six-atomic fragment of an aromatic molecule from the cited work $;^{3}$ for convenience it is reproduced in Fig. 1. For the sake of simplicity in the theoretical treatment we assume all the interatomic distances to be the same, say $D$. Following Whiffen et $a l^{3,20}$ also all interbond angles are assumed to be equal, viz. $120^{\circ}$. Under these conditions we can define two out-plane bendings ( $c f$. Fig. 1$)^{24}$

$$
\begin{aligned}
& \gamma=D^{-1}\left(-3 z_{1}+z_{2}+z_{3}+z_{4}\right) \\
& \gamma^{\prime}=D^{-1}\left(z_{1}-3 z_{4}+z_{5}+z_{6}\right)
\end{aligned}
$$

and the two torsions:

$$
\begin{aligned}
& \delta=2 D^{-1} 3^{-\frac{1}{2}}\left(-2 z_{1}+z_{2}+2 z_{4}-z_{5}\right) \\
& \delta^{\prime}=2 D^{-1} 3^{-\frac{1}{2}}\left(2 z_{1}-z_{3}-2 z_{4}+z_{6}\right)
\end{aligned}
$$

Here the coordinates are expressed in terms of the out-of-plane cartesian displacements. ${ }^{25}$ Scully and

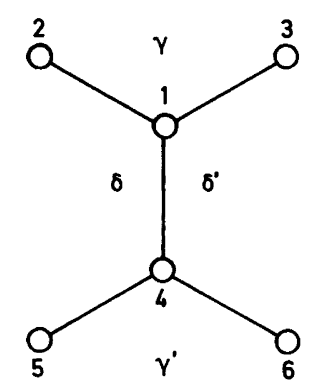

Fig. 1. Numbering of atoms and valence coordinates for out-of-plane vibrations of a sixatomic fragment of an aromatic molecule.

Whiffen ${ }^{20}$ have introduced the two types of outof-plane coordinates (i) deformation and (ii) twisting.

(i) The two deformation coordinates in the sixatomic fragment (Fig. 1) appear to be identical to the out-of-plane bendings ${ }^{24} \gamma$ and $\gamma^{\prime}$.

(ii) Define the combination of torsions

$\varepsilon=2^{-\frac{1}{2}}\left(\delta+\delta^{\prime}\right)=2 D^{-1} 6^{-\frac{1}{2}}\left(z_{2}-z_{3}-z_{5}+z_{6}\right)$

This coordinate appears to be proportional to Whiffen's twisting.

Consider two sets of coordinates pertaining to the six-atomic structure of Fig. 1: (a) $\mathbf{S}_{\mathrm{a}}=\left\{\gamma, \gamma^{\prime}, \varepsilon\right\}$, referred to as Whiffen's coordinates on one hand, and (b) $\mathbf{S}_{\mathbf{b}}=\left\{\gamma, \gamma^{\prime}, \delta, \delta^{\prime}\right\}$ on the other. In case (a) the coordinates are independent while a redundancy occurs in the set (b). The latter set (b) consists of conventional valence coordinates ${ }^{24}$ only. Scully and Whiffen ${ }^{20}$ have argued, with reference to previous works, ${ }^{3,26}$ that the interaction constant between $\gamma$ and $\varepsilon$ is much smaller than between $\gamma$ and $\delta$. We shall find a sound theoretical basis for the validity of this statement.

The simplest force field in terms of Whiffen's coordinates, $\mathbf{S}_{\mathbf{a}}$, is given by

$\mathbf{F}=\left[\begin{array}{lll}F_{\gamma} & 0 & 0 \\ & F_{\gamma} & 0 \\ & & F_{\varepsilon}\end{array}\right]$

where we have assumed $F_{\gamma \gamma}=0$ in addition to $F_{\gamma \varepsilon}=0$. The $\gamma$ and $\gamma^{\prime}$ coordinates are assumed to be symmetrically equivalent for the sake of simplicity. The most general harmonic force field in terms of $\mathrm{S}_{\mathrm{b}}$ is given by 
$\mathbf{f}=\left[\begin{array}{cccc}f_{\gamma} & f_{\gamma \gamma} & f_{\gamma \delta} & -f_{\gamma \delta} \\ & f_{\gamma} & -f_{\gamma \delta} & f_{\gamma \delta} \\ & & f_{\delta} & f_{\delta \delta} \\ & & & f_{\delta}\end{array}\right]$

where we have assumed that also $\delta$ and $\delta^{\prime}$ are symmetrically equivalent. We have deduced the transformation matrix of $\mathrm{S}_{\mathrm{b}}=\mathbf{T S}_{\mathrm{a}}$ and used it to find the connection between the two force constant matrices according to ${ }^{23}$

\section{$F=T^{\prime} f T$}

The result is

$$
\mathbf{F}=\left[\begin{array}{lll}
A & B & 0 \\
& A & 0 \\
& & C
\end{array}\right]
$$

where

$A=f_{\gamma}+\frac{4}{3} 3^{\frac{1}{2}} f_{\gamma \delta}+\frac{2}{3}\left(f_{\delta}-f_{\delta \delta}\right)$

$B=f_{\gamma \gamma}-\frac{4}{3} 3^{\frac{1}{2}} f_{\gamma \delta}-\frac{2}{3}\left(f_{\delta}-f_{\delta \delta}\right)$

$C=f_{\delta}+f_{\delta \delta}$

The zeroes in the above matrix $\mathbf{F}$ are equivalent to $F_{y \varepsilon}=0$. They are not additional approximations, but emerge as a consequence of the symmetry restrictions. This part of the analysis constitutes a theoretical basis of the statement of Scully and Whiffen ${ }^{20}$ referred to above.

Next we introduce as an approximation $F_{y y}=$ $B=0$. This requirement may be fulfilled in infinitely many ways because of the presence of redundancy in the $\mathbf{S}_{\mathrm{b}}$ coordinates. Perhaps the simplest way to meet the requirement is to put

$f_{\gamma \gamma}=f_{\gamma \delta}=0$

$f_{\delta \delta}=f_{\delta}$

In conclusion we have obtained a guide for a simple force field approximation: (1) Define two torsions for every CC bond, corresponding to $\delta$ and $\delta^{\prime}$. This requirement implies the definition of "outer" torsions of the types HCCH and HCCC in addition to the "inner" CCCC. (2) Assume a considerable interaction term between two such torsions, in fact of the same order of magnitude as the main torsional force constant. (3) All other interaction terms may tentatively be neglected. (4) Main force constants are also attributed to $\mathrm{CH}$ and $\mathrm{CC}$ out-ofplane bendings. We adopt the convention to include one out-of-plane bending for every $\mathrm{C}$ atom. In every case the three surrounding atoms (of which one may be an $\mathrm{H}$ ) move in one direction, while the given central atom, $\mathrm{C}$, moves in the opposite direction. If all interbond angles are assumed to be equal $\left(120^{\circ}\right)$ this convention gives a prescription for an unambiguous selection of coordinates of this type. Even if we use accurate structural data with angles deviating from $120^{\circ}$ we may assume that the ambiguities in the construction of the approximate force field are insignificant.

\section{BENZENE}

Coulson and Senent ${ }^{4}$ have compared their calculated out-of-plane vibrational frequencies for benzene with experimental data from 1936 through 1946. In Table 1 these calculated frequencies (column a) are compared with modern experimental data. ${ }^{27}$ Coulson and Senent used two frequencies $\left(E_{1 g}\right.$ and $\left.A_{2 u}\right)$ to adjust their two parameters of the force field. These two frequencies happen to be very close to the modern experimental data. Otherwise the agreement is found to be unsatisfactory. Our calculated frequencies from the previous force field with three out-of-plane parameters ${ }^{13}$ are seen to be still worse in most of the cases; cf. Table 1 (column b). The calculations of Whiffen ${ }^{3}$ are also included in the table (column c). In the original paper they too were compared with old experimental data (1936 -46). Nevertheless they do show a satisfactory agreement with the modern data. We have mentioned the comparatively large number of parameters as a disadvantage in Whiffen's approximation. In the present work we have reduced the number of parameters substantially without significant loss in the accuracy of the approximation.

Guided by the prescription of the theoretical considerations we assigned a force constant to every $\delta(\mathrm{CCCC})$ and $\delta^{\prime}(\mathrm{HCCH})$ torsion. Several trials were made, including those with different numerical values for $\delta$ and $\delta^{\prime}$. A final set was produced, however, with only one parameter $f_{\delta}$ pertaining to both types of the torsions. An 
Table 1. Calculated and observed frequencies $\left(\mathrm{cm}^{-1}\right)$ for the out-of-plane vibrations of benzene.

\begin{tabular}{lrllll}
\hline Species & $\mathrm{a}^{a}$ & $\mathrm{~b}$ & $\mathrm{c}$ & $\mathrm{d}$ & Obs. $^{27}$ \\
\hline$B_{2 g}$ & 1110 & 1238 & 992.2 & 985 & 990 \\
& 638 & 438 & 705.9 & 650 & 707 \\
$E_{1 g}$ & 850 & 741 & 851.1 & 831 & 846 \\
$A_{2 u}$ & 671 & 686 & 673.0 & 686 & 673 \\
$E_{2 u}$ & 1049 & 981 & 984.0 & 968 & 967 \\
& 413 & 319 & 404.2 & 398 & 398 \\
\hline
\end{tabular}

${ }^{a} \mathrm{a}$, Two parameters. ${ }^{4} \mathrm{~b}$, Three parameters. ${ }^{13} \mathrm{c}$, Seven parameters. ${ }^{3} \mathrm{~d}$, Three parameters; present work.

Table 2. Calculated and observed frequencies $\left(\mathrm{cm}^{-1}\right)$ for the out-of-plane vibrations of naphthalene.

\begin{tabular}{lrrrll}
\hline Species & \multicolumn{1}{c}{$\mathrm{a}^{a}$} & $\mathrm{~b}$ & $\mathrm{c}$ & $\mathrm{d}$ & Obs. $^{\mathbf{1 3 , 2 1}}$ \\
\hline$B_{1 \boldsymbol{g}}$ & 1040 & 934 & 920 & 941 & 943 \\
& 722 & 702 & 704 & 744 & 717 \\
& 365 & 312 & 365 & 400 & 386 \\
$B_{2 g}$ & & & & & \\
& 1107 & 1305 & 971 & 982 & 980 \\
& 937 & 952 & 881 & 889 & 876 \\
& 687 & 679 & 770 & 745 & $446 / 778$ \\
$A_{\boldsymbol{u}}$ & 436 & 379 & 485 & 476 & \\
& 1143 & 1150 & 1022 & 976 & 970 \\
& 917 & 776 & 807 & 860 & 841 \\
& 549 & 417 & 594 & 596 & 581 \\
$B_{3 u}$ & 222 & 185 & 207 & 206 & 195 \\
& 1053 & 999 & 962 & 967 & 958 \\
& 776 & 714 & 759 & 772 & 782 \\
& 422 & 429 & 445 & 432 & 476 \\
\hline
\end{tabular}

${ }^{a}$ a, Two parameters. ${ }^{4}$ b, Three parameters. ${ }^{13} \mathrm{c}$, Seven parameters. ${ }^{21} \mathrm{~d}$, Five parameters; present work.

interaction term between $\delta$ and the corresponding $\delta^{\prime}$ is prescribed to be essential. Our final value is even somewhat larger than the principal term $f_{\delta}$. The final values (in mdyne/ $\AA$ ):

$f_{\delta}=0.05$

$f_{\delta \delta}=0.07$

$f_{\gamma}=0.2$

The calculated values from this exceedingly simple force field are listed in column $d$ of Table 1 . The agreement with the observed frequencies are seen to be comparable with those of Whiffen (column c). The only discrepancy of some seriousness occurs for our low $B_{2 g}$ frequency, but does not violate the good picture in general. The crucial test for the success of our force field, of course, is the possible transferability of the numerical parameters to other aromatic molecules.

\section{NAPHTHALENE}

The computational results of Coulson and Senent $^{4}$ for the out-of-plane frequencies of naphthalene are shown in Table 2 (column a), where they are compared with modern experimental data. For the two lowest $A_{u}$ frequencies and the lowest $B_{1 g}$ frequency the calculations show much better agreement with these modern data than is the case with the best 
available assignments from the time when the calculations were published. ${ }^{4}$ In general the calculations, using only two parameters, are amazingly accurate for their time (1955). The accuracy is not satisfactory, however, according to modern standards. Neither is it the case for the three-parameter approximation ${ }^{13}$ given in column b. Column c shows the calculations of Scully and Whiffen. ${ }^{20,21}$ In the most recent ones of these papers $^{21}$ a computational error affecting the $A_{u}$ frequencies has been corrected. These calculations (column c) according to the Whiffen approximation, are seen to give an acceptable agreement with the observed frequencies. The results of the present approximation (column d) are seen to be comparable to those from the Whiffen approximation. The agreement is definitely not worse. To be precise, our approximation gives a better agreement for eight frequencies and worse for seven. The observed frequencies given in Table 2 are adopted from the assignment of Krainov, ${ }^{28}$ also quoted elsewhere. ${ }^{13,29}$ This assignment is compatible with the one of Scully and Whiffen ${ }^{21}$ except for one of the $B_{2 g}$ frequencies $\left(778 \mathrm{~cm}^{-1}\right)$, which is included as an alternative assignment in Table 2.

In the present computation (column d) the three force constants from benzene (see above) were employed with unchanged numerical values. For the two out-of-plane bendings where only $\mathrm{C}$ atoms participate we applied the value (in mdyne/ $/ \AA$ ) ${ }^{13}$

$f_{\gamma^{\prime}}=0.15$

This type does not exist in benzene. For the two torsions of the middle bond (where $\delta$ and $\delta^{\prime}$ are symmetrically equivalent) the benzene value of $f_{\delta}$ $=0.05 \mathrm{mdyne} / \AA$ was found to be as good as any. A substantial improvement was achieved by lowering the $\delta / \delta^{\prime}$ interaction around the middle bond to:

$f_{\delta \delta^{\prime}}=0.02$

In this way we ended up with five parameters for naphthalene. The number is still smaller than the number of parameters in Whiffen's approximation.

\section{FURTHER APPLICATIONS}

We wish to mention that the present approximation also was applied to anthracene and pyrene, the additional molecules which have been treated by the method of Whiffen et al..$^{3,20}$ We are not giving any details of those results here. In general they confirm the success of the fiveparameter approximation. The numerical values were all transferred from the benzene and naphthalene applications without introducing any new types of constants.

Acknowledgement. Financial support to BNC from The Norwegian Research Council for Science and the Humanities is gratefully acknowledged.

\section{REFERENCES}

1. Wilson, E. B., Jr. Phys. Rev. 45 (1934) 706.

2. van den Bossche, M. and Manneback, C. Ann. Soc. Sci. Bruxelles 54-B (1934) 230.

3. Whiffen, D. H. Philos. Trans. R. Soc. London A 248 (1955) 131.

4. Coulson, C. A. and Senent, S. J. Chem. Soc. (1955) 1813.

5. Scherer, J. R. and Overend, J. Spectrochim. Acta 17 (1961) 719.

6. Scherer, J. R. J. Chem. Phys. 36 (1962) 3308.

7. Califano, S. Ric. Sci. 3 (1963) 461.

8. Neto, N., Scrocco, M. and Califano, S. Spectrochim. Acta 22 (1966) 1981.

9. Schettino, V., Neto, N. and Califano, S. J. Chem. Phys. 44 (1967) 2724.

10. Neto, N. and di Lauro, C. Spectrochim. Acta 26 $A$ (1970) 1175.

11. Schettino, V. J. Mol. Spectrosc. 34 (1970) 78.

12. Bree, A., Kydd, R. A., Misra, T. N. and Vilkos, V. V. B. Spectrochim. Acta A 27 (1971) 2315.

13. Whitmer, J. C., Cyvin, S. J. and Cyvin, B. N. Z. Naturforsch. Teil A 33 (1978) 45.

14. Bakke, A., Cyvin, B. N., Whitmer, J. C., Cyvin, S. J., Gustavsen, J. E. and Klaeboe, P. Z. Naturforsch. Teil A 34 (1979) 579.

15. Cyvin, S. J., Cyvin, B. N., Brunvoll, J., Whitmer, J. C., Klaeboe, P. and Gustavsen, J. E. Z. Naturforsch. Teil A 34 (1979) 876.

16. Cyvin, S. J., Cyvin, B. N. and Brunvoll, J. Z. Naturforsch. Teil A 34 (1979) 887.

17. Cyvin, S. J., Brunvoll, J., Cyvin, B. N. and Mastryukov, V. S. Z. Naturforsch. Teil A 34 (1979) 1512.

18. Ohno, K. J. Mol. Spectrosc. 72 (1978) 238.

19. Ohno, K. J. Mol. Spectrosc. 77 (1979) 329.

20. Scully, D. B. and Whiffen, D. H. J. Mol. Spectrosc. 1 (1957) 257.

21. Scully, D. B. and Whiffen, D. H. Spectrochim. Acta 16 (1960) 1409.

22. Evans, D. J. and Scully, D. B. Spectrochim. Acta 20 (1964) 891. 
23. Cyvin, S. J. In Cyvin, S. J., Ed., Molecular Structures and Vibrations, Elsevier, Amsterdam 1972, Chapter 6.

24. Wilson, E. B., Jr., Decius, J. C. and Cross, P. C. Molecular Vibrations, McGraw-Hill, New York 1955.

25. Cyvin, B. N., Cyvin, S. J. and Müller, A. Acta Chem. Scand. 23 (1969) 1352.

26. Bell, R. P. Trans. Faraday Soc. 41 (1945) 293.

27. Brodersen, S. and Langseth, A. K. Dan. Vidensk. Selsk. Mat. Fys. Skr. 1 (1956) No. 1.

28. Krainov, E. P. Opt. Spektrosk. 16 (1964) 763.

29. Hagen, G. and Cyvin, S. J. J. Phys. Chem. 72 (1968) 1446.

Received April 18, 1980. 